\title{
Change in Ultrasonic Parameters and Dislocation Structures during Fatigue Process of Aluminum Alloy under High Stress Amplitude
}

\author{
Xiaohua Min ${ }^{1}$,*, Hiroshi Kato ${ }^{1}$, Fuxing Yin $^{2}$ and Seiji Konuma ${ }^{3}$ \\ ${ }^{1}$ Department of Mechanical Engineering, Faculty of Engineering, Saitama University, Saitama 338-8570, Japan \\ ${ }^{2}$ Steel Research Center, National Institute for Materials Science, Tsukuba 305-0047, Japan \\ ${ }^{3}$ Material Characterization Laboratory, Kanagawa High-Technology Foundation, Kanagawa Science Park, Kawasaki 213-0012, Japan
}

Aluminum alloy (A2024-T3) specimens were used for a fatigue testing by subjecting them to a stress amplitude of $150 \mathrm{MPa}$. At different numbers of the fatigue cycles, specimens were removed from the fatigue tester, and then subjected to the ultrasonic measurement, the dislocation density measurement, the hardness testing, and so on. From the Fourier spectrum of the bottom echo, the peak intensity (PI), the peak frequency (PF) and the average gradient of the transfer function (AGTF) were obtained. The dislocation density was obtained by the X-ray diffraction analysis. AGTF, the dislocation density and the hardness decreased at the initial stage, and then gradually increased with the increasing number of the fatigue cycles. PI showed a tendency to increase as fatigue cycles increased, but no change occurred in PF. The change of ultrasonic parameters in the fatigue process was quantitatively discussed according to Granato and Lücke dislocation-string model. Then the in-process ultrasonic measurement was carried out in the fatigue testing of the aluminum alloy by using the water bag to obtain ultrasonic parameters. PI and AGTF rapidly increased at the initial stage, and then gradually increased with increasing number of the fatigue cycles. These results suggest that the dislocation density steeply increased at the initial stage of the fatigue process, and then they gradually increased.

(Received December 17, 2004; Accepted April 7, 2005; Published June 15, 2005)

Keywords: nondestructive evaluation, aluminum alloy, fatigue process, ultrasonic parameters, dislocation density, hardness, Granato and Lücke model

\section{Introduction}

In past decades, numerous studies have been carried out on changes in microstructures of metallic materials in the fatigue process, and have shown that the density and arrangement of dislocations change successively with cyclic loadings. ${ }^{1-10)}$ Since mechanical properties of materials are largely influenced by the microstructures and if these changes are evaluated nondestructively, it is possible to monitor the fatigue damaging process of products and constructions under service conditions. From this viewpoint, the ultrasonic measurement has been carried out in the fatigue process. Bratina $^{11)}$ and Joshi ${ }^{12)}$ reported that the attenuation coefficient had three stages during the fatigue testing of steel and aluminum: a slight increase at an early stage, a long stable period and rapid increase in the final stage. The initial increase was caused by the increase in the dislocation density, and the terminal increase was attributed to appearance of microcracks and their subsequent growth. Recently, non-contact ultrasonic measurement methods, such as the electromagnetic acoustic resonance (EMAR) method ${ }^{13-17)}$ and the laser ultrasonic method, ${ }^{18)}$ have been developed to perform highly sensitive and reliable ultrasonic measurement during the fatigue process. Hirao et al. ${ }^{13-15)}$ measured the attenuation coefficient and the sound velocity in the fatigue testing by using the EMAR method and found that the attenuation coefficient showed a maximum at a certain period of the fatigue life. Although the EMAR method and the laser ultrasonic method have lots of merit, they also have a disadvantage that the apparatus is complex and high in cost.

The authors ${ }^{19)}$ developed the ultrasonic measurement method using a water bag to measure ultrasonic parameters in aluminum alloy plates during the fatigue testing, and

*Graduate Student, Saitama University reported that a peak intensity of the Fourier spectrum of the bottom echo and an average gradient of the transfer function (AGTF) periodically changed with the fatigue cycle, and that an average of cyclic change in AGTF gradually increased as the number of the fatigue cycles increased. ${ }^{20)}$ In the fatigue process, the ultrasonic parameters change following change in the microstructure, especially the dislocation structure, and it is vital to clarify a relationship between the ultrasonic parameters and the dislocation structures in the fatigue process for nondestructive evaluation of the degradation of materials suffering cyclic loadings.

In the present work, the fatigue testing was carried out with a high stress amplitude of $150 \mathrm{MPa}$ for aluminum alloy (A2024-T3) plates, and specimens were subjected to the ultrasonic measurement, the dislocation density measurement and so on. Then relationships between the ultrasonic parameters and the dislocation density were discussed according to Granato-Lücke dislocation-string model. ${ }^{21,22)}$ The ultrasonic measurement using the water bag was also carried out in-process during fatigue testing to examine the changes in the ultrasonic parameters in the fatigue process.

\section{Experimental Procedures}

\subsection{Specimen preparation and fatigue testing}

Specimens with slightly curved sides were fabricated from aluminum alloy (A2024-T3) plates in commercial use, as shown in Fig. 1, so that the specimen axis was parallel to the rolling direction. The chemical composition is tabulated in Table 1. The alloy plates had been strengthened by T3 treatment: solution treatment, quenching, cold working, and then natural aging successively. Mechanical properties of the alloy were $328 \mathrm{MPa}$ in yield strength $(0.2 \%$ offset proof stress), $465 \mathrm{MPa}$ in tensile strength and $21 \%$ in elongation.

Specimens were subjected to a fatigue testing under 


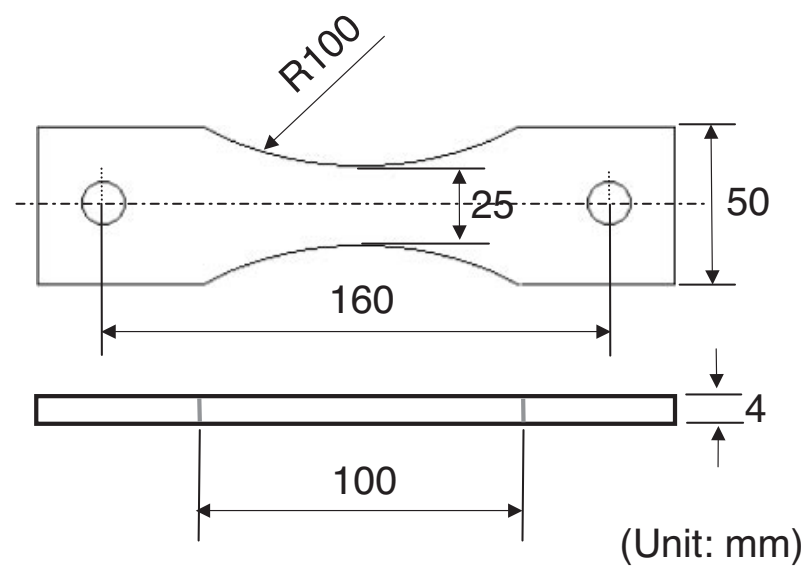

Fig. 1 Shape and dimensions of specimen for fatigue testing.

Table 1 Chemical composition of A2024.

(mass\%)

\begin{tabular}{cccccccccc}
\hline $\mathrm{Cu}$ & $\mathrm{Mg}$ & $\mathrm{Mn}$ & $\mathrm{Si}$ & $\mathrm{Fe}$ & $\mathrm{Cr}$ & $\mathrm{Zn}$ & $\mathrm{Zr}+\mathrm{Ti}$ & $\mathrm{Ti}$ & $\mathrm{Al}$ \\
\hline 4.62 & 1.53 & 0.56 & 0.06 & 0.18 & 0.01 & 0.19 & 0.03 & 0.02 & Bal.
\end{tabular}

conditions of a stress ratio of 0.05 (a tension-tension type), a frequency of $10 \mathrm{~Hz}$ and a stress amplitude of $150 \mathrm{MPa}$ (the expected fatigue life: $1 \times 10^{5}$ cycles). After a required number of the fatigue cycles, they were removed from the fatigue tester for various measurements and observations. Some of the specimens were subjected to fatigue testing to fracture with the in-process ultrasonic measurement.

\subsection{Ultrasonic measurement and wave analysis}

The ultrasonic measurement was carried out with the conventional immersion method by using a transducer generating a longitudinal wave of $20 \mathrm{MHz}$ in frequency with a focal distance of $25.4 \mathrm{~mm}$ in water. The ultrasonic wave was measured with a water path (a distance between the transducer and the specimen) of about $7 \mathrm{~mm}$. Under this condition, the ultrasonic wave was focused at a reverse surface. Ultrasonic waves reflected from obverse and reverse surfaces (hereafter referred to as surface and bottom echoes, respectively) were recorded in a digital storage with a sampling time of $0.2 \mathrm{~ns}$. A typical wave is shown in Fig. 2. Bottom echoes were subjected to the fast Fourier transformation (FFT) analysis to obtain the Fourier spectrum. A peak intensity (PI) and a peak frequency (PF) of the Fourier spectrum of the bottom echo shown in Fig. 3 were obtained, but no change was obtained for PF. Therefore, in the present paper, only results of PI were shown.

The following transfer function $\Gamma(\omega)$ was also obtained.

$$
\Gamma(\omega)=20 \log _{10}\left(\frac{f(\omega)}{f_{0}(\omega)}\right)
$$

where $f_{0}(\omega)$ and $f(\omega)$ are the bottom echo spectra before and in the fatigue testing, respectively. A typical transfer function is shown in Fig. 4. As shown in the figure, an average gradient of the transfer function (AGTF) was obtained in a frequency range of $14.5-30.5 \mathrm{MHz}$. In the previous works, ${ }^{23,24)}$ the transfer function was measured for plastically

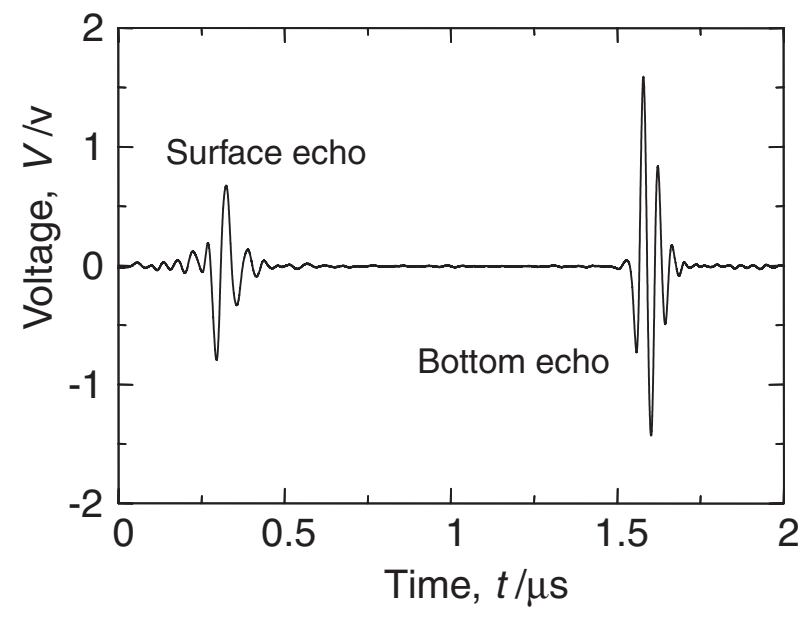

Fig. 2 Typical surface and bottom echoes.

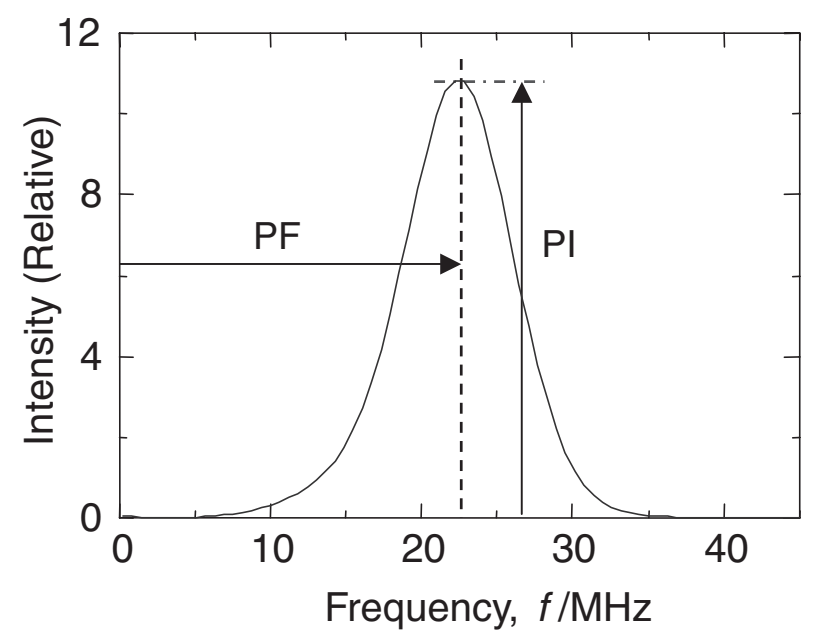

Fig. 3 Definition of peak intensity (PI) and peak frequency (PF) of Fourier spectrum.

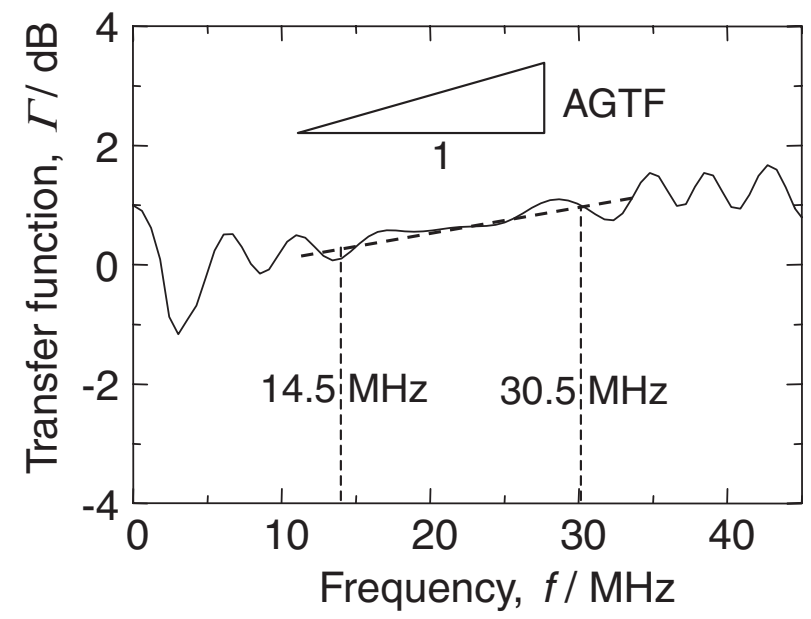

Fig. 4 Typical transfer function and average gradient of transfer function (AGTF).

deformed specimens, and it was found that AGTF showed a monotonic change with plastic strain, ${ }^{24)}$ which means that AGTF is related to structures, such as crystal grains, dislocations, and so on. In the present work, the change in 


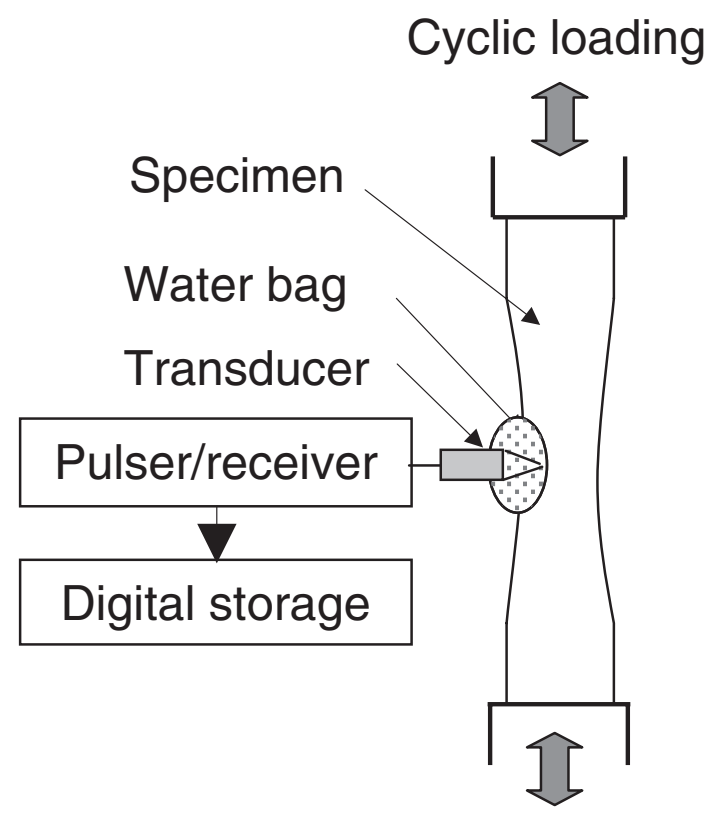

(a) Schematic representation

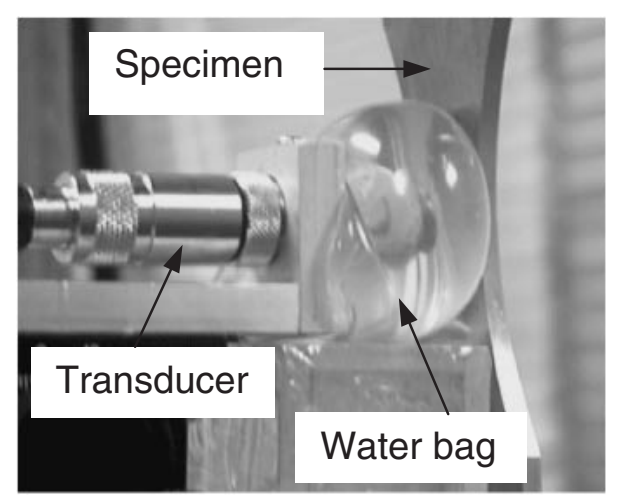

(b) Photograph

Fig. 5 Setup for in-process ultrasonic measurement during fatigue testing.

the Fourier spectrum or the ultrasonic waveform was mainly affected by the dislocation structures, and hence AGTF was measured and compared with the dislocation structures.

Figure 5 shows a setup for in-process ultrasonic measurement during fatigue testing. The ultrasonic measurement was carried out with the specimen kept at an average stress of the fatigue testing $(166 \mathrm{MPa})$. The transducer was encapsulated in a water bag made of latex membrane with a thickness of $69 \mu \mathrm{m}$, and was set at a center of the specimen (hereafter this method was referred to as the local immersion method). A generating frequency of the transducer was $20 \mathrm{MHz}$ and a focal distance was $25.4 \mathrm{~mm}$ in water. In this measurement, the ultrasonic wave was focused on a reverse surface of the specimen to obtain a larger bottom echo. Surface and bottom echoes were recorded in a digital storage with a sampling time of $0.2 \mathrm{~ns}$.

\subsection{Measurement of dislocation density and observation of microstructures}

For measurement of the dislocation density by the X-ray diffraction analysis, pieces of $15 \mathrm{~mm}$ in length, $10 \mathrm{~mm}$ in width and $4 \mathrm{~mm}$ in thickness were sectioned at the center of specimens, polished to a thickness of $2 \mathrm{~mm}$ and finished by electro-polishing. Diffraction profiles of (111), (200), (220), (222) and (311) planes were measured with the X-ray diffractometer at $40 \mathrm{kV}$ and $250 \mathrm{~mA}$. After background subtraction, Lorentz-polarization-absorption correction and normalization, the dislocation density was estimated by using the modified Warren-Averbach X-ray diffraction profile analysis. $^{25-28)}$

Microstructures were observed through transmission electron microscope (TEM) at $200 \mathrm{kV}$. Thin pieces were cut at the center of the specimen normal to the specimen axis, polished to a thickness of $0.1 \mathrm{~mm}$, and suffered an electropolishing of $15 \mathrm{~V}$ and $2.8 \mathrm{~A}$ in Nital at $249 \mathrm{~K}$. Then the TEM observation was carried out parallel to the specimen axis.

\subsection{Measurement of hardness and surface roughness}

The Vickers hardness was measured with a load of $49 \mathrm{~N}$ at 10 positions for each specimen. The surface roughness of the specimen was measured with a laser surface roughness meter at a center of the specimen.

\section{Results}

Figure 6 shows changes in ultrasonic parameters with the number of the fatigue cycles. In the figure, PI was normalized by the peak intensity before fatigue testing. PI was largely

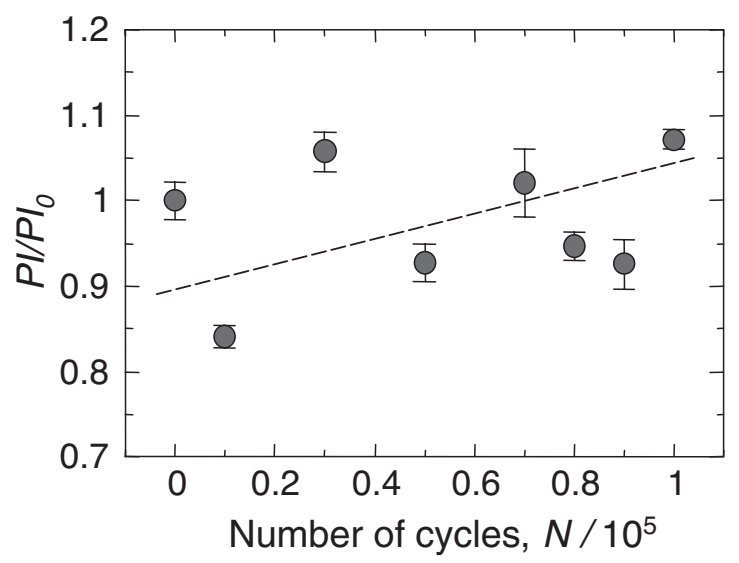

(a) Peak intensity normalized by initial peak intensity $\left(P I / P I_{0}\right)$

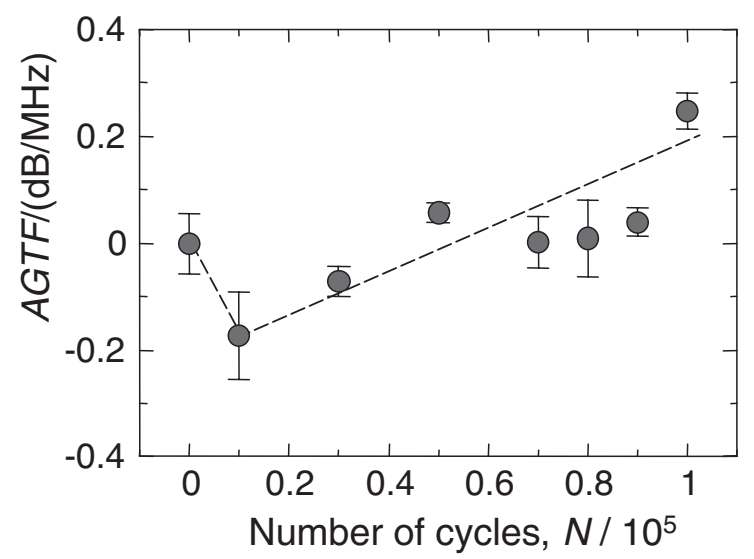

(b) Average gradient of transfer function (AGTF)

Fig. 6 Change in ultrasonic parameters with number of fatigue cycles. 


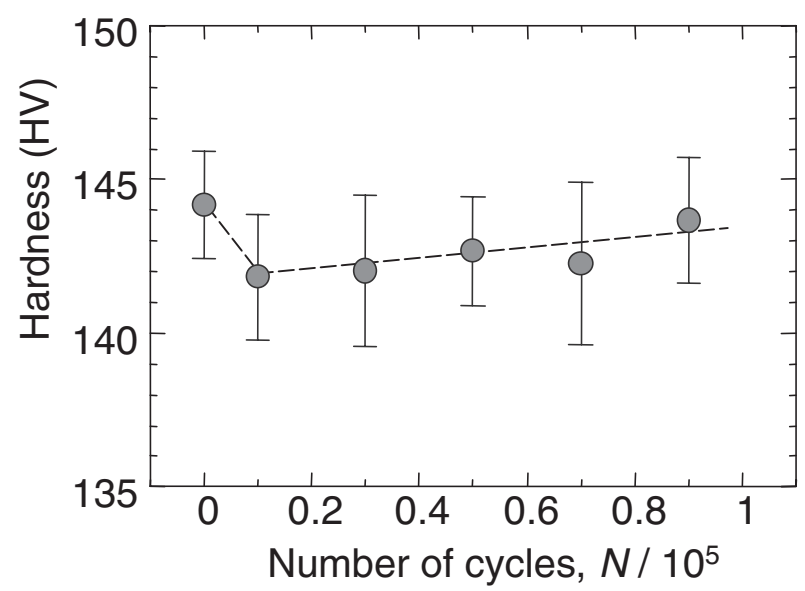

Fig. 7 Change in Vickers hardness with number of fatigue cycles.

scattered but showed a tendency to increase as the number of the fatigue cycles increased. AGTF decreased at an initial stage, and then gradually increased with increasing number of the fatigue cycles.

As shown in Fig. 7, the Vickers hardness also decreased at the initial stage, and then gradually increased.

Typical TEM micrographs obtained at the center of the specimen are shown in Fig. 8. In the figures, the dark areas are clusters of fragmented dislocations. A considerable number of dislocations existed in the specimen before fatigue testing, and the dislocation arrangement did not clearly change in the fatigue process to form cell walls, which were observed in the previous works. ${ }^{13-15)}$ The TEM observation was carried out for different crystal grains and positions, but no large difference was observed. In the present work, the dislocation density was higher before fatigue testing, and TEM observation was performed with higher magnification than that in the previous works. ${ }^{13-15)}$ These differences may have caused the difference in the observed dislocation structures.

The dislocation density was obtained with the X-ray diffraction analysis as shown in Fig. 9. Before fatigue testing, the specimen had a high dislocation density of $3.6 \times 10^{13} /$ $\mathrm{m}^{2}$. In the fatigue testing, the dislocation density decreased at the initial stage, and then gradually increased with increasing number of the fatigue cycles. The change in the dislocation density in the fatigue process was similar to AGTF and the hardness.

\section{Discussion}

\subsection{Influence of factors on ultrasonic parameters}

Figure 10 shows a schematic representation of the propagation of the ultrasonic wave in the specimen. First, the ultrasonic wave is irradiated from a transducer, and then the wave is incident upon the surface of a specimen. At the surface, some ratio of the wave is reflected with a reflectivity determined by acoustic impedances of water and the specimen. The remains pass through the obverse surface and propagate in the specimen with scattering at microstructures, such as dislocations, grain boundaries, precipitates, fine cracks, and so on. Figure 11 shows a typical microstructure in the aluminum alloy plates. In the figure, crystal grains were elongated in the rolling direction (vertical in the figure). Dark spots in the figure are coarse precipitates of about $10 \mu \mathrm{m}$ in size. The ultrasonic wave is reflected from the reverse surface of the specimen and then returns to the obverse surface. In consideration of the traveling path of the ultrasonic wave, the intensity $I$ of the bottom echo is given by

$$
I=I_{0} R_{\mathrm{S}}(f) R_{\mathrm{M}}(f) R_{\mathrm{B}}(f)
$$

where $I_{0}$ is the intensity of the incident wave. $R_{\mathrm{S}}(f)$ and $R_{\mathrm{B}}(f)$ are the reducing factors relating to the reflectivity and the roughness of the obverse and reverse surfaces, respectively. $R_{\mathrm{M}}(f)$ is the reducing factor due to scattering at microstructures. And, $R_{\mathrm{S}}(f), R_{\mathrm{B}}(f)$ and $R_{\mathrm{M}}(f)$ are functions of the wave frequency.

In the present work, the surface roughness took a constant value of about $0.12 \mu \mathrm{m}$ in the fatigue process except at the terminal stage, as shown in Fig. 12, and hence the factors $R_{\mathrm{S}}(f)$ and $R_{\mathrm{B}}(f)$ can be assumed to be constant. And also, in the fatigue process, the grain size and the distribution of precipitates were kept almost constant. Since the ultrasonic measurement was finished before crack appearance, the effect of the crack on the intensity of the ultrasonic wave can be neglected from $R_{\mathrm{M}}(f)$. Finally, the dislocation density and structure remained as variables to affect the ultrasonic wave intensity $I$. In the following section, changes in the ultrasonic parameters are discussed from evolution of the dislocation density and arrangement.

\subsection{Relation between ultrasonic parameters and dislo- cation density}

In the previous reports, ${ }^{13,14,29)}$ the attenuation coefficient showed a maximum at a certain period of fatigue life. These results were obtained by using heat-treated specimens of lower dislocation density. However, the present work was carried out by using specimens that underwent the T3 treatment before fatigue testing and containing higher dislocation density. Therefore dislocations behaved in the fatigue process differently from the previous reports. In this section, changes in the ultrasonic parameters in the fatigue process are discussed.

First, a relation between the attenuation coefficient and the dislocation density is discussed. According to the dislocation damping theory, ${ }^{21)}$ a magnitude of damping is classified by a parameter $D$ given by

$$
D=\frac{2 \pi f_{0} A}{B}
$$

where $f_{0}$ is the resonant frequency calculated to be $870 \mathrm{MHz}$ for A2024. $A\left(=\pi \rho b^{2}\right)$ is the effective mass per unit dislocation loop length, where $\rho$ is the density and $b$ is the Burgers vector. $B$ is the damping constant calculated to be $9.6 \times 10^{-4} \mathrm{~kg} \cdot \mathrm{m}^{-1} \mathrm{~s}^{-1}$ for A2024 by using the Leibfried's formula. ${ }^{21)}$ Substituting these values in eq. (3), the value of $D$ was obtained to be 0.41 , which was close to a value of pure aluminum of $0.39 .^{22}$ ) The damping in the present case was classified to be a large damping, and the maximum loss occurred at a frequency $f_{\mathrm{m}}\left(=D f_{0}\right)$ calculated to be $357 \mathrm{MHz}$. Since a frequency of $20 \mathrm{MHz}$ used in the present work is far lower than $f_{\mathrm{m}}$, the sound velocity $V$ and the 

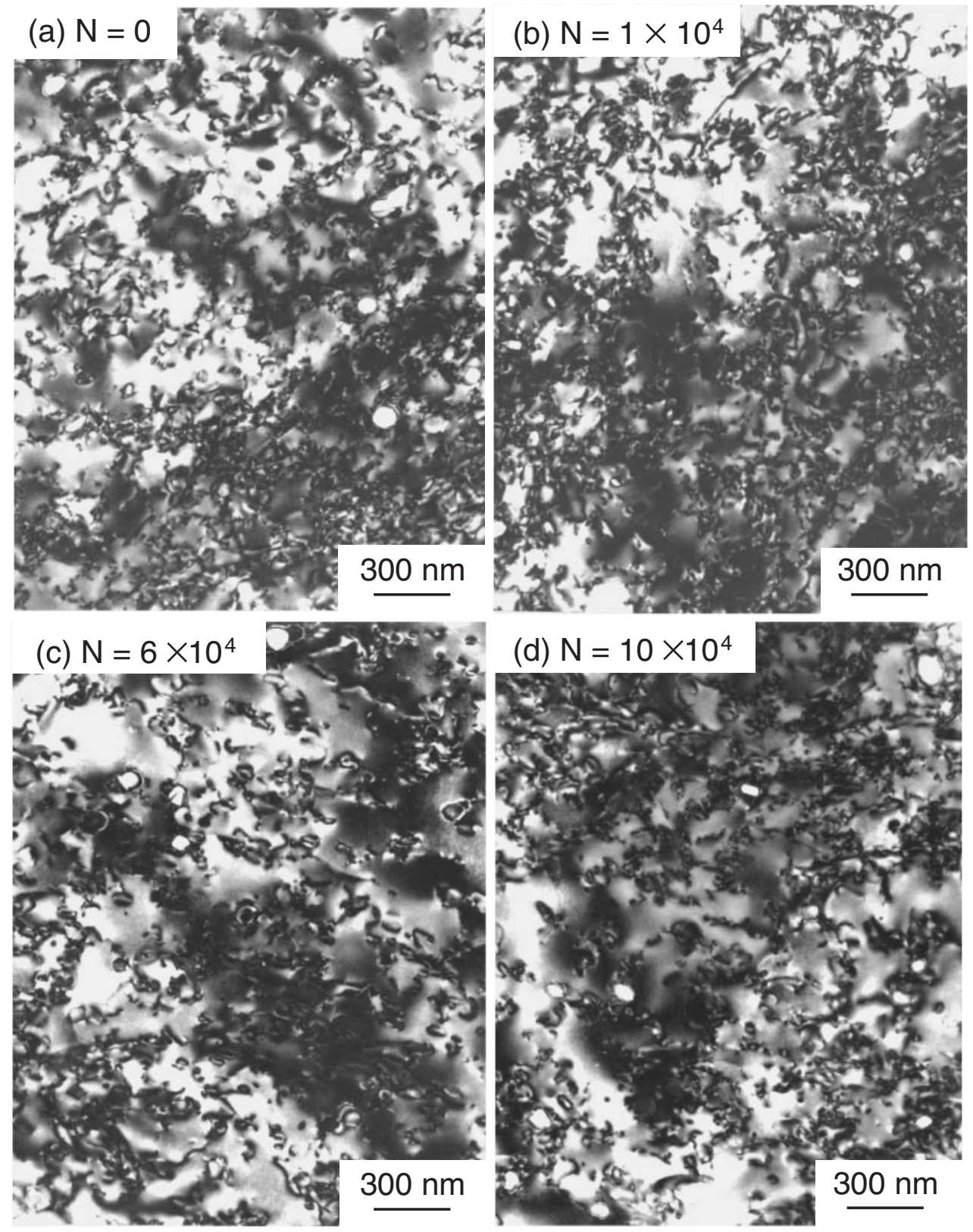

Fig. 8 TEM micrographs of specimens suffered different numbers of fatigue cycles.

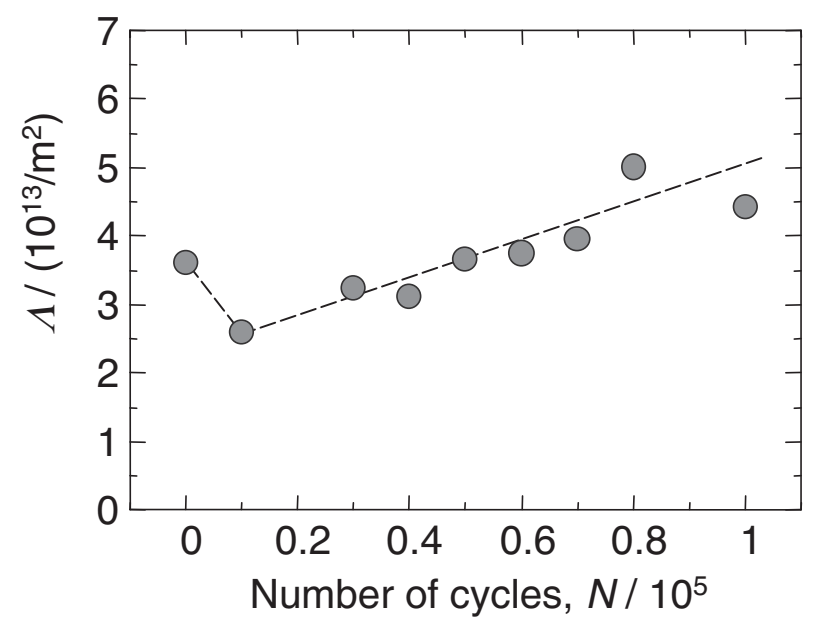

Fig. 9 Change in dislocation density $(\Lambda)$ with number of fatigue cycles. attenuation coefficient $\alpha$ are given by ${ }^{13,27)}$

$$
\begin{aligned}
\frac{\left(V-V_{0}\right)}{V_{0}} & =-\left(\frac{4 G b^{2}}{\pi^{4} C}\right) \Lambda L \\
\alpha & =\left(\frac{16 G B b^{2}}{\pi^{4} C^{2}}\right) \Lambda L^{4} f^{2}
\end{aligned}
$$

where $\Lambda$ is the dislocation density, $L$ is the dislocation loop length, and $C$ is the line tension of the dislocation given by $2 G b^{2} / \pi(1-v)$, where $G$ and $v$ are the shear modulus $(27.3 \mathrm{GPa})$ and the Poisson's ratio $(0.343)$, respectively.

The sound velocity $V$ was measured with the sing-around method $^{30)}$ as shown in Fig. 13. Substituting the sound velocity and the dislocation density in eq. (4), the dislocation loop length $L$ was obtained as shown in Fig. 14. In the figure, the dislocation loop length decreased from $0.99 \mu \mathrm{m}$ with increasing number of the fatigue cycles. Substituting the measured dislocation density $\Lambda$ and the dislocation loop 


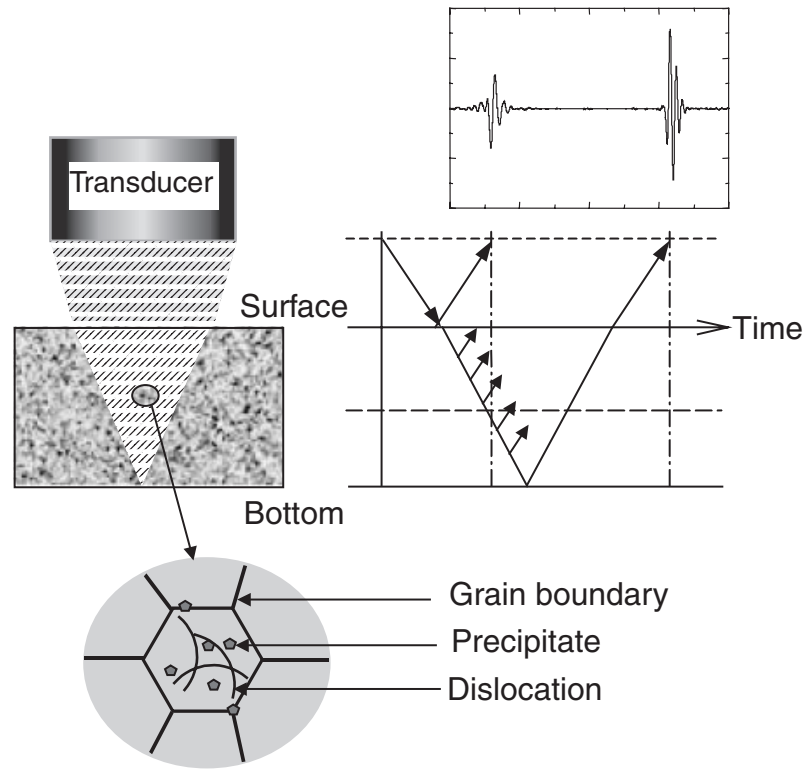

Fig. 10 Schematic representation of propagation of ultrasonic wave in specimen.

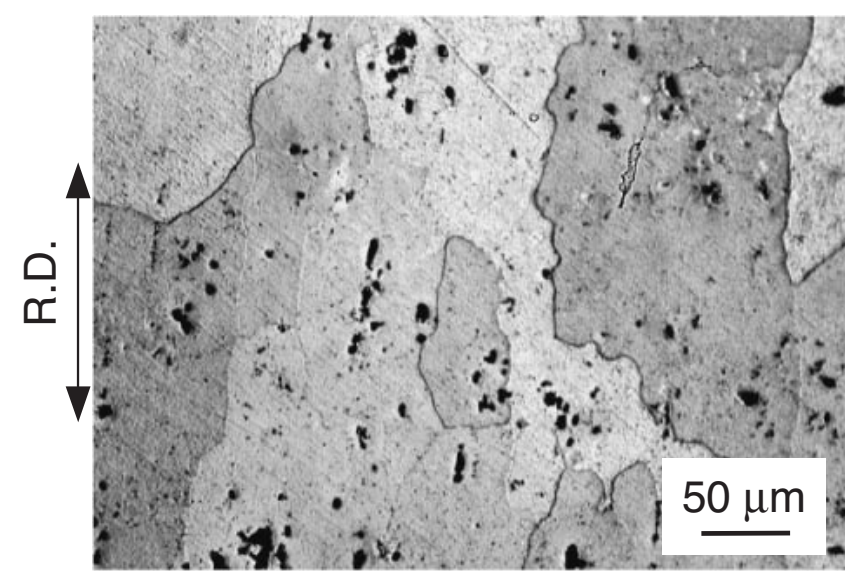

Fig. 11 Optical micrograph of specimen. The rolling direction (R.D.) is vertical in the figure.

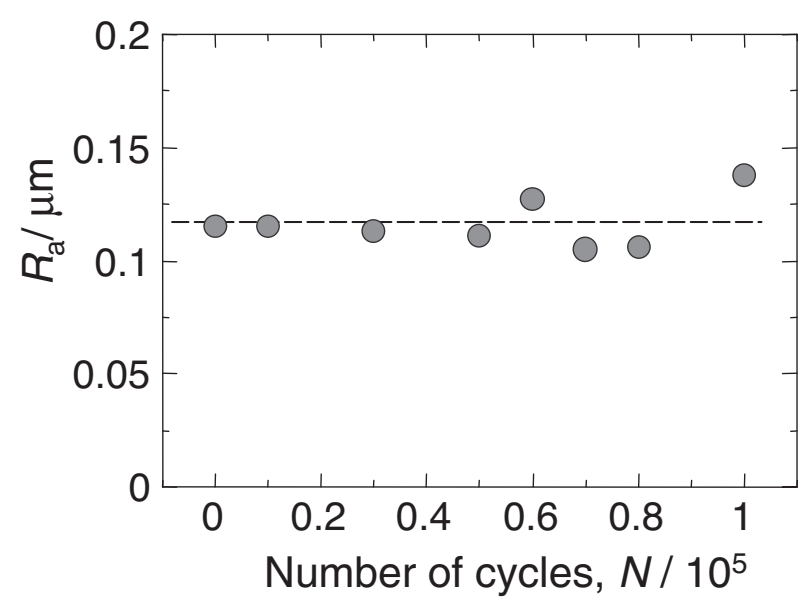

Fig. 12 Change in surface roughness $\left(R_{\mathrm{a}}\right)$ with number of fatigue cycles.

length $L$ in eq. (5), the attenuation coefficient $\alpha$ was estimated as shown in Fig. 15. In the figure, the attenuation coefficient decreased with increasing number of the fatigue

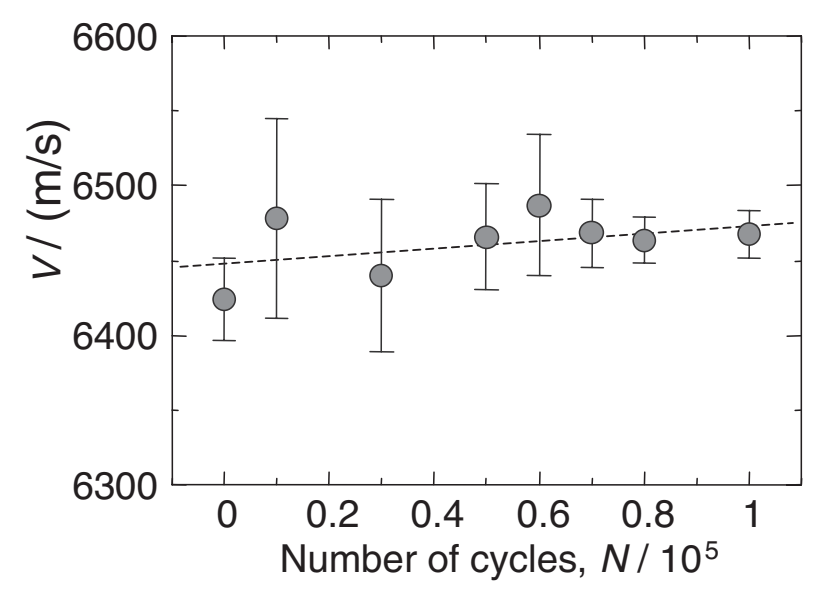

Fig. 13 Change in sound velocity $(V)$ with number of fatigue cycles.

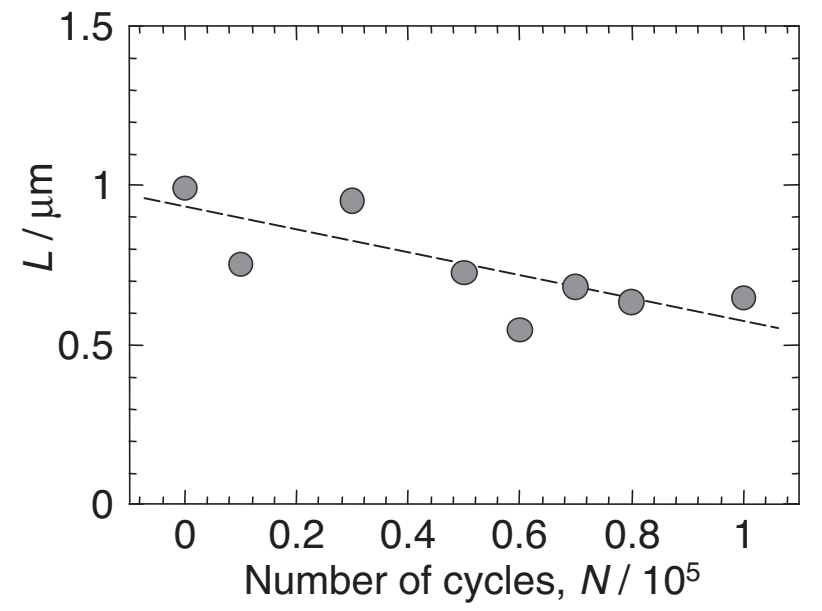

Fig. 14 Change in dislocation loop length $(L)$ with number of fatigue cycles.

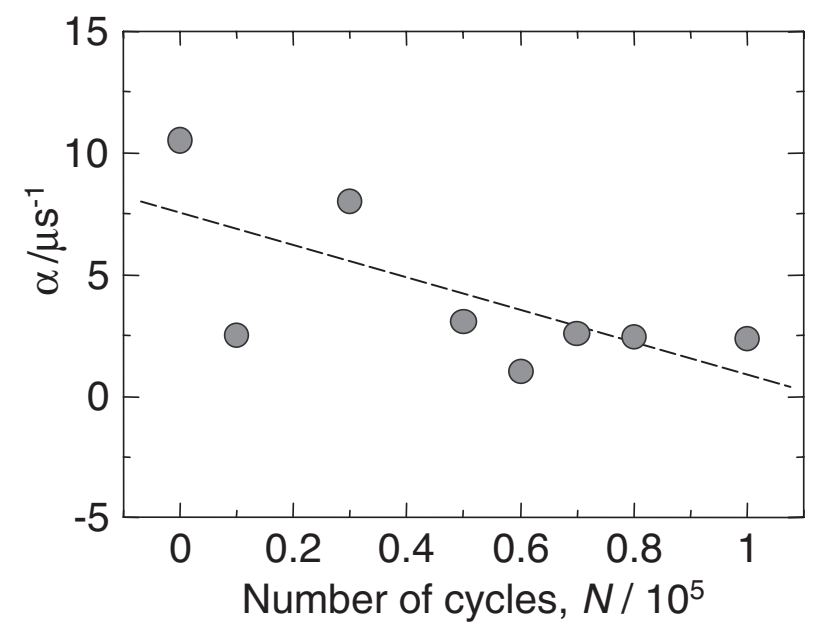

Fig. 15 Change in attenuation coefficient $(\alpha)$ with number of fatigue cycles.

cycles, which tendency was in good agreement with PI being increased with increasing number of the fatigue cycles.

Then, a change in AGTF in the fatigue process is discussed. ${ }^{20)}$ Assuming that the ultrasonic wave is reduced during traveling in the specimen due to scattering at 
microstructures, and that the Fourier spectrum is changed as follows

$$
f(\omega)=f_{0}(\omega)(1-\delta \omega) m,
$$

the gradient of the transfer function $(d \Gamma(\omega) / d \omega)$ is given by,

$$
\frac{d \Gamma(\omega)}{d \omega}=-\frac{20}{\ln 10} \delta
$$

where, $f_{0}(\omega)$ and $f(\omega)$ are the Fourier spectra of the ultrasonic wave before and in the fatigue testing, and $m(\leq 1)$ and $\delta(\ll 1)$ are the parameters relating to the attenuation coefficient. Since AGTF increased with increasing number of the fatigue cycles as shown in Fig. 6(b), the parameter $\delta$ should be decreased following eq. (7) to result in the decrease in the attenuation coefficient.

It is thought that the dislocation loop length changes in the fatigue process similar to the dislocation density. As shown in Fig. 14, however, the dislocation loop length did not show a transient change at the initial stage of the fatigue process, but decreased monotonically. This was because the sound velocity used in the calculation was largely scattered at the early stage of the fatigue process and did not follow the transient change. Hence the dislocation loop length did not show the transient change at the early stage. The attenuation coefficient also showed the monotonic change because it was obtained by using dislocation loop length.

\subsection{In-process ultrasonic measurement during fatigue testing}

To understand the fatigue damaging process, it is desirable to evaluate the dislocation density and arrangement inprocess during the fatigue testing. In the above section, it was found that the ultrasonic parameters can be used as measures for evaluation of the dislocation density. Therefore, ultrasonic parameters were measured in-process in the fatigue testing, as shown in Fig. 16. The ultrasonic parameters rapidly increased at an early stage, and then gradually increased with increasing number of the fatigue cycles. These results indicate that the dislocation density steeply increased at the early stage, and then gradually increased to a critical value.

The ultrasonic parameters obtained by the in-process measurement were differently changed in the fatigue process from those obtained by the off-line measurement because the stress state was different in both ultrasonic measurements. In the case of the off-line measurement, the measurement was carried out with specimens removed from the fatigue tester. Therefore, no stress was applied to the specimens. Before fatigue testing, the specimen contained the high dislocation density. In the fatigue test, the specimen suffered the tensile stress in the different direction from the cold working, and tangled dislocations were released in the early stage of the fatigue testing to result in the decrease in the dislocation density. Then with increasing number of the fatigue cycles, the dislocations were tangled again to increase the dislocation density. Following the change in the dislocation density, the ultrasonic parameters once decreased in the early stage, and then gradually increased. To the contrary, in the case of the in-process measurement, the ultrasonic measurement was carried out with the specimen kept at the average stress of the

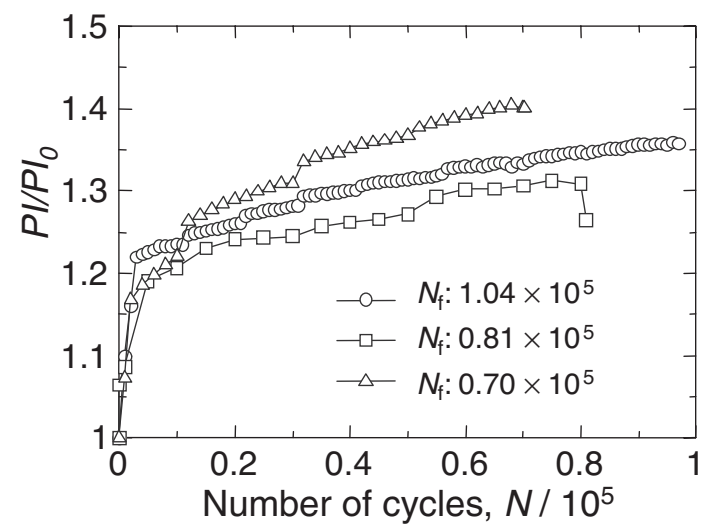

(a) Peak intensity normalized by initial peak intensity $\left(P I / P I_{0}\right)$

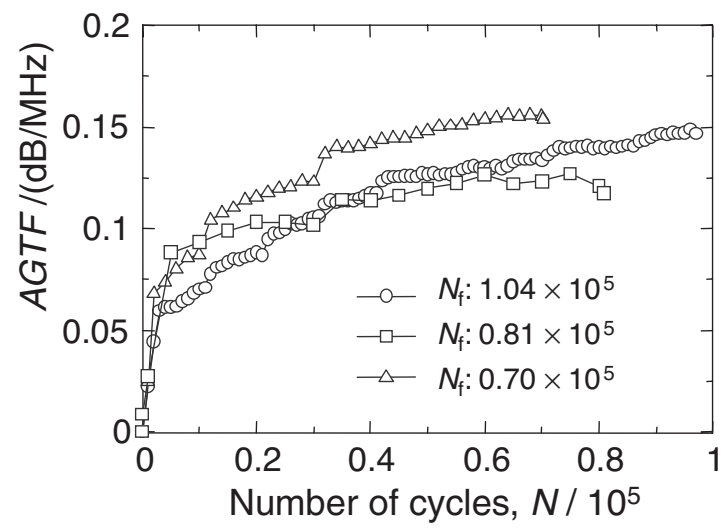

(b) Average gradient of transfer function (AGTF)

Fig. 16 Change in ultrasonic parameters obtained in-process measurement with number of fatigue cycles.

loading cycle, and many dislocations were kept tangled or trapped under a tensile stress state. Therefore, the high dislocation density was not reduced but steeply increased at the early stage to result in the monotonic increase in the ultrasonic parameters.

\section{Conclusions}

The fatigue testing with a stress amplitude of $150 \mathrm{MPa}$ was carried out with aluminum alloy A2024-T3 specimens. At the required number of the fatigue cycles, the specimen was removed from the fatigue tester and then subjected to the ultrasonic measurement. The dislocation density and the Vickers hardness were also obtained. The average gradient of the transfer function (AGTF), the dislocation density and the hardness decreased at the initial stage, and then gradually increased with increasing number of the fatigue cycles. The peak frequency did not change in the fatigue process. The change in the ultrasonic parameters in the fatigue process was calculated according to the Granato-Lücke dislocation model, and was in good agreement with the tendency of the measured ultrasonic parameters.

The ultrasonic measurement of aluminum alloy specimens was carried out in-process during the fatigue testing by the local immersion method using the water bag. The peak intensity (PI) and AGTF rapidly increased at the early stage of the fatigue testing, and then gradually increased with 
increasing number of the fatigue cycles. These changes suggest that the dislocation density increased at the early stage, and then gradually increased to a critical value in the fatigue process. It was also found that the local immersion method using the water bag is a useful tool for in-process ultrasonic measurement.

\section{Acknowledgements}

The authors would like to thank Dr. K. Kageyama, Department of Mechanical Engineering, Saitama University, for discussion with him on the relation between ultrasonic parameters and microstructures.

\section{REFERENCES}

1) K. U. Snowden: Acta Metall. 11 (1963) 675-684.

2) C. E. Feltner and C. Laird: Acta Metall. 15 (1967) 1633-1653.

3) J. K. Lee and C. Laird: Mater. Sci. Eng. 54 (1982) 53-64.

4) H. F. Chai and C. Laird: Mater. Sci. Eng. 93 (1987) 159-174.

5) D. Y. Ye, X. Y. Tong, L. J. Yao and X. X. Yin: Mater. Chem. Phys. 56 (1998) 199-204.

6) B. Gong, Z. Wang and Z. G. Wang: Acta Mater. 47 (1999) 317-324.

7) T. Fujii, C. Watanabe, Y. Nomura, N. Tanaka and M. Kato: Mater. Sci. Eng. A 319-321 (2001) 592-596.

8) Y. El-Madhoun, A. Mohamed and M. N. Bassim: Mater. Sci. Eng. A 359 (2003) 220-227.

9) G. F. Dirras, J.-L. Duval and W. Swiatnicki: Mater. Sci. Eng. A 263 (1999) 85-95.
10) C. Watanabe, T. Fujii, S. Onaka and M. Kato: Mater. Sci. Eng. A 319321 (2001) 506-510.

11) W. J. Bratina: Physical Acoustic vol. 3 (A), ed. By W. P. Mason, (Academic Press, New York, Massachusetts, 1966), pp. 223-291.

12) N. R. Joshi: Ultrasonic Detection of Fatigue Damage, Ph.D. Thesis, (The Johns Hopkins University, Baltimore, Maryland, 1973).

13) M. Hirao, H. Ogi, N. Suzuki and T. Ohtani: Acta Mater. 48 (2000) 517524.

14) H. Ogi, T. Hamaguchi and M. Hirao: J. Alloys Comp. 310 (2000) 436439.

15) M. Hirao, H. Ogi and H. Yasui: NDT\&E Int. 34 (2001) 179-183.

16) W. Johnson: Mater. Sci. Eng. A 309-310 (2001) 69-73.

17) W. Johnson: J. Alloys Comp. 310 (2000) 423-426.

18) S. Luxenburger and W. Arnold: Ultrasonics 40 (2002) 797-801.

19) X. H. Min, H. Kato, H. Saito and K. Kageyama: J. JSNDI 52 (2003) 697-702. (In Japanese)

20) X. H. Min and H. Kato: Mater. Sci. Eng. A 372 (2004) 269-277.

21) A. Granato and K. Lücke: J. Appl. Phys. 27 (1956) 583-593.

22) A. Granato and K. Lücke: J. Appl. Phys. 27 (1956) 789-805.

23) L. M. Shen and H. Kato: NDT\&E Int. 32 (1999) 355-361.

24) H. Kato, N. Itoi and K. Kageyama: J. JSNDI 50 (2001) 34-40. (in Japanese)

25) F. Yin, T. Hanamura, O. Umezawa and K. Nagai: Mater. Sci. Eng. A 354 (2003) 31-39.

26) T. Ungár, S. Ott, P. G. Sanders, A. Borbély and J. R. Weertman: Acta Mater. 46 (1998) 3693-3699.

27) T. Ungár, M. Victoria, P. Marmy, P. Hanák and G. Szenes: J. Nucl. Mater. 276 (2000) 278-282.

28) T. Ungár: Mater. Sci. Eng. A 309-310 (2001) 14-22.

29) S. Kenderian, T. P. Berudt, R. E. Green, Jr. and B. B. Djordjevtic: Mater. Sci. Eng. A 348 (2003) 90-99.

30) J. Krautkrämer and H. Krautkrämer: Ultrasonic Testing of Materials, (Springer-Verlag, Berlin, 1990) p. 236. 